\title{
High-Order Wavelet Reconstruction for Multi-Scale Edge Aware Tone Mapping
}

\author{
Alessandro Artusi ${ }^{\mathrm{a}}$, Zhuo Su ${ }^{\mathrm{b}, \mathrm{c}}$, Zongwei Zhang's ${ }^{\mathrm{b}, \mathrm{c}}$, Dimitris Drikakis ${ }^{\mathrm{d}}, \mathrm{Xianan}_{\mathrm{Luo}}{ }^{\mathrm{b}, \mathrm{c}}$ \\ ${ }^{a}$ Graphics and Imaging Laboratory Department of Informatics and Applied Mathematics University of Girona. \\ ${ }^{b}$ National Engineering Research Center of Digital Life, Guangzhou, China. \\ ${ }^{c}$ School of Information Science and Technology, Sun Yat-sen University, Guangzhou, China. \\ ${ }^{d}$ Fluid Mechanics and Computational Science Cranfield University.
}

\begin{abstract}
This paper presents a High Order Reconstruction (HOR) method for improved multi-scale edge aware tone mapping. The study aims to contribute to the improvement of edge-aware techniques for smoothing an input image, while keeping its edges intact. The proposed HOR methods circumvent limitations of the existing state of the art methods, e.g., altering the image structure due to changes in contrast; remove artefacts around edges; as well as reducing computational complexity in terms of implementation and associated computational costs. In particular, the proposed method aims at reducing the changes in the image structure by intrinsically enclosing an edge-stop mechanism whose computational cost is comparable to the state-of-the-art multi-scale edge aware techniques.
\end{abstract}

Keywords:

\section{Introduction}

2 High Order Reconstruction (HOR) methods, intro3 duced by Harten et al. [1], have been used exten4 sively for solving the hyperbolic conservation laws and 5 the Hamilton-Jacobi equations [2]. Additionally, these 6 methods have been applied to image processing (image 7 compression), denoising [3] and segmentation [4]. Due 8 to their ability to reduce oscillations around function 9 discontinuities, these methods can be potentially used 10 as an edge aware interpolation tool. Edge-aware tech${ }_{11}$ niques such as anisotropic diffusion [5], bilateral filter12 ing $[6,7]$ and neighborhood filtering rely on sophisti${ }_{13}$ cated type of spatially varying kernels. Often, they tend 14 to either generate artificially staircasing effects or ring15 ing effects around sharp edges [8]. These artifacts can ${ }_{16}$ be reduced using a post-processing step at the price of 17 increasing the computational cost and the number of pa18 rameters used [9]. To have better control of the details ${ }_{19}$ over the spatial scale, one can apply edge-aware tech${ }_{20}$ niques in a multi-scale fashion. However, the bilateral ${ }_{21}$ filtering is inappropriate for multi-scale detailed decom${ }_{22}$ position [10]. Other edge-aware techniques that sup${ }_{23}$ port the multi-scale approach $[10,11,9]$ also encompass 24 some flaws, e.g., they are not able to achieve a plausible ${ }_{25}$ reproduction of all important image features [12] and ${ }_{26}$ may change the image structure.

${ }_{27}$ Therefore, there is a need to develop methods that are
${ }_{28}$ reducing as much as possible any change into the image ${ }_{29}$ structure without increasing the complexity or compu${ }_{30}$ tational cost.

${ }_{31}$ In this paper, we link the edge-aware problem to the 32 typical problem of interpolation. In particular, we proз3 pose a novel wavelet scheme that uses a robust predictor ${ }_{34}$ operator, based on the HOR method, which intrinsically 35 encloses an edge-stop mechanism to avoid influence of ${ }_{36}$ pixels from both sides of an edge. To have a better con${ }_{37}$ trol of details over the spatial scale, we employ the HOR ${ }_{38}$ method in conjunction with a multi-scale scheme.

${ }_{39}$ We demonstrate the usability of the proposed method to ${ }_{40}$ solve a typical problem in the context of High Dynamic ${ }_{41}$ Range (HDR) imaging, called tone mapping as defined ${ }_{42}$ in Banterle et al. [13].

43 The approach is formulated as follows; we decom${ }_{44}$ pose an input HDR image, making use of wavelet de45 composition and through the use of HOR methods sep${ }_{46}$ arate its coarse and fine features (details). The coarse 47 and fine features are then manipulated to achieve the de${ }_{48}$ sired tone and details levels. Finally, the output image ${ }_{49}$ is reconstructed. The advantage of the above approach 50 is that it does not require the introduction of any edge${ }_{51}$ stopping function that limits possible image-structure 52 changes.

53 To understand this concept, Figure 1 shows the dis54 tortion map as output of the Dynamic Range Indepen- 

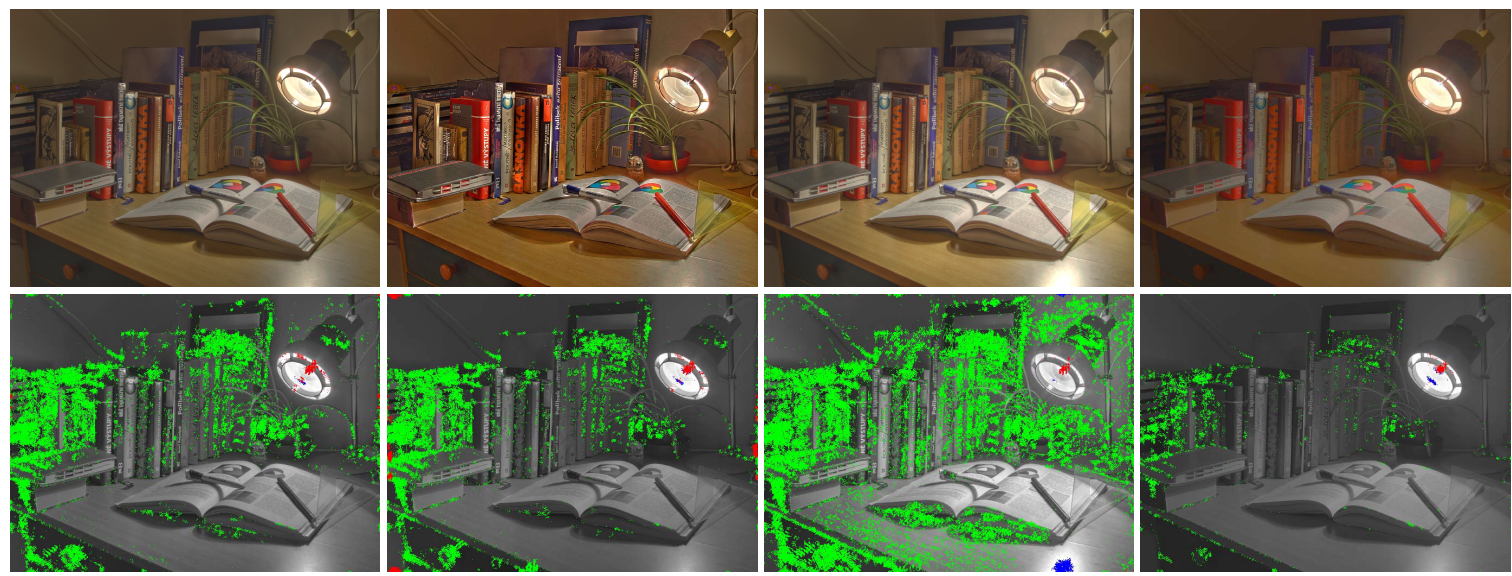

Figure 1: Comparison of the state-of-the-art multiscale edge aware based tone mapping operators and the present HOR: $1^{\text {st }}$ row: output of the various techniques. $2^{\text {nd }}$ row: distortion map of the DRIM metric [12]. This map is showing the pixels that shows a distorsion with $95 \%$ of probability to been seen by the Human Visual System $(H V S)$. Blue pixels are areas where invisible contrast is introduced; red pixels are areas where reversal of visible contrast is noticeable and green pixels shows areas of lost of contrast. The map is showing of a reduction of more than $50 \%$ of the pixels affected by loss of contrast when the the HOR method is used. Parameters used - Farbmann et al. [10] multiscale approach balanced - Fattal's [11] $\alpha=0.9, \beta=0.16$ and $\gamma=0.8$ - Paris et al. [9] $\sigma_{r}=\log (2.5), \alpha=0.5$ and $\beta=0.0$ (for conveying the local effect) - The Present HOR $\beta=0.7, \gamma=0.9$.

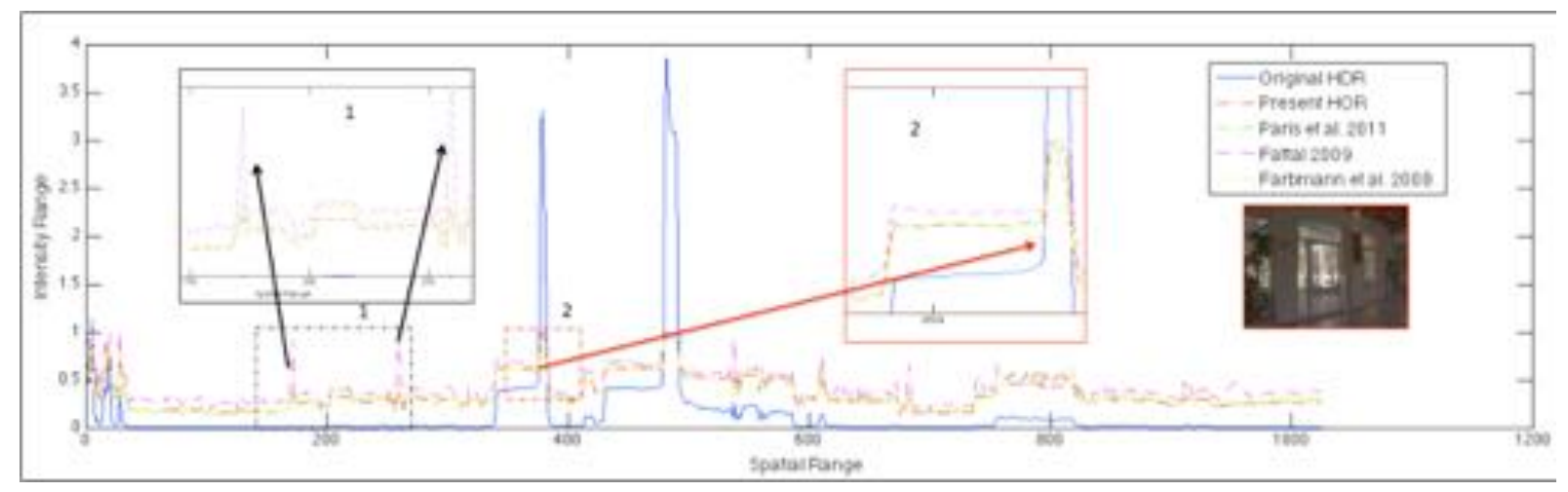

Figure 2: Intensity profile for the tone mapping operators on an HDR mage for line 300: The $1^{\text {st }}$ zoomed area, clearly shows how Fattal's [11] method (undesirably) increases the intensity profile to the maximum value of 1 . In the $2^{\text {nd }}$ zoomed area (Paris et al. [9] green line), the intensity profile is modified. 
${ }_{55}$ dent metric (DRIM) introduced by Aydin et al. [12] for ${ }_{56}[10,11,9]$ and the technique proposed in this paper. The ${ }_{57}$ original HDR image is used as reference, and the output 58 of the tone mapping operator is compared to it. A cer${ }_{59}$ tain amount of lost of contrast (green) is clearly visible, 60 and this may change the overall image structure [12]. ${ }_{61}$ The map shows that using the present HOR reduces the 62 number of pixels affected by loss of contrast by more 63 than $50 \%$.

${ }_{64}$ Moreover, the intensity profile may change as shown 65 in Figure 2. The Fattal method [11] may have an un${ }_{66}$ desirable increase of the intensity profile to the maxi${ }_{67}$ mum output value 1 ( $1^{\text {st }}$ zoomed area). The structure of 68 the original profile may be undesirably modified (green 69 line) as shown for the method [9] ( $2^{\text {nd }}$ enlarged area).

${ }_{70}$ These methods may result in prohibitive computational ${ }_{71}$ costs (see Paris et al. [9]). An efficient implementa72 tion [14] of the method presented by Paris et al. [9] ${ }_{73}$ is also discussed in Section 6.

74 The proposed approach retains the same advantages 75 introduced by the traditional edge aware approaches 76 such as Paris et al. [9], and Fattal [11], namely with re${ }_{77}$ spect to obtaining local properties and providing robust 78 smoothing, hence avoiding the use of pixels from both 79 sides of the edge. The main contributions of this work 80 can be summarized as follows:

81 1. Establish a link between the robust smoothing concept to the reconstruction problem of a nonsmoothed function.

2. Achieve a complex solution of the edge-aware problem, through a simple and flexible point-wise manipulation by using HOR method.

3. Propose an edge-aware filter that produces halo free results; reduces the changes in the image structure as defined by the DRIM metric and its computational cost is increasing linearly with respect to the number of the input pixels $N$.

\section{2. Related Work}

\section{${ }_{93}$ Edge Aware Filters}

${ }_{94}$ Edge aware techniques are used to smooth an image 95 while keeping its edges intact, preventing pixels located ${ }_{96}$ on one side of a strong edge from influencing pixels on ${ }_{97}$ the other side. This concept can be used to separate high 98 frequency information from low frequency information 99 such as texture and details. Once this separation is pe100 formed the high and low frequencies information can be 101 independently manipulated and re-composed.

${ }_{102}$ In the past, techniques able to preserve edges $[6,8,5]$ ${ }_{103}$ have been applied to image manipulation $[15,16,17$,
104 11]. These techniques produce acceptable results, but 105 often introduce visible ringing effects arising from the 106 Poisson equation [15] and filtering, as discussed in [10, 1078 . Moreover, they need several parameters, that are im108 age dependent, making their set-up difficult for practi${ }_{109} \mathrm{cal}$ applications [17]. Our approach offers a solution, 110 that produces results at least as good as the above tech111 niques, runs linearly in time with respect to the number 112 of the input pixels and is not dependent on a large num113 ber of parameters.

\section{${ }_{114}$ Multi-Scale Edge Aware Filters}

115 Recently, several edge-aware techniques that can be 116 used in the multi-scale framework, have been presented. ${ }_{117}$ Typically, these methods exploit the multi-scale ap118 proach by making use of pyramid mechanisms such as ${ }_{119}$ Laplacian [18], Gaussian [19], and Wavelets [20].

${ }_{120}$ The Laplacian approach, in the context of edge-aware, 121 has been recently revised by Paris et al. [9] through the 122 use of local transformation which makes the Laplacian ${ }_{123}$ approach suitable for edge-aware operations. Farbman ${ }_{124}$ et al. [10] employed the weighted least square to build 125 an alternative edge preserving operator and extend it to 126 multi-scales as well. Fattal et al. [15] used the Gaus127 sian Pyramid to compress the high dynamic range of the 128 input image, followed by the full image reconstruction ${ }_{129}$ through the use of the Poisson solver.

${ }_{130}$ The aforementioned techniques share with our ap${ }_{131}$ proach the multi-scale 'philosophy', but are using dif${ }_{132}$ ferent methods such as the Laplacian [10,9] and Gaus${ }_{133}$ sian [15] pyramids. Moreover, they are based on the so134 lution of a linear system [10], a Poisson solver [15], or 135 bilateral filtering all of which generate artifacts around ${ }_{136}$ edges [8]. Li et al. [21] proposed a multi-scale approach ${ }_{137}$ based on wavelets where each sub-band signal is mod${ }_{138}$ ified using a gain map that controls the local contrast. ${ }_{139}$ Fattal [11] presented an edge avoiding technique based ${ }_{140}$ on a second generation wavelet. Our approach inte${ }_{141}$ grates within the wavelet mechanism a HOR technique 142 that does not require any edge-stop function for com${ }_{143}$ puting a large set of weights in the interpolation step 144 as in [11]. Consequently, using the present approach 145 there is no need for any particular precaution against 146 the strong edges and distortions of the image structure 147 are reduced.

\section{${ }_{148}$ 3. Background}

149 Fixed stencil approximation techniques, such as ${ }_{150}$ piecewise linear and cubic interpolation, are often used 151 to reconstruct the missing points of a function. These 152 methods are working well in the case where the func${ }_{153}$ tion is smooth; however, if the function is only piece- 


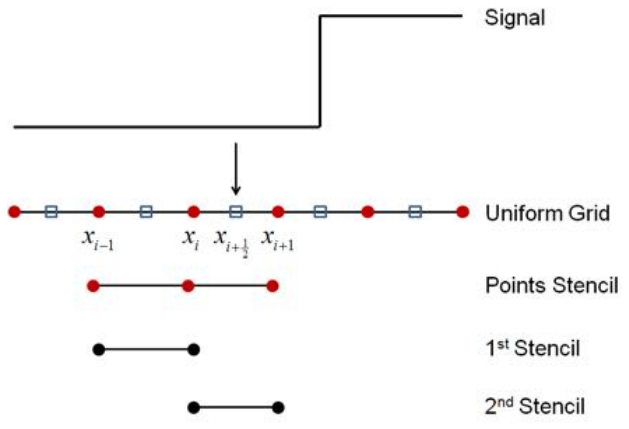

Figure 3: Example of the HOR scheme mechanism. (Top row) The original staircase signal. ( $2^{\text {nd }}$ row) The uniform grid points: (circle red) input points, (square blue) points to be interpolated. ( $3^{\text {rd }}$ row) The stencil points used by the HOR scheme. ( $4^{\text {th }}$ and $5^{\text {th }}$ rows): Two separated stencils used to define the two interpolants by the HOR scheme.

154 wise smooth the fixed stencil approximation may not be 155 adequate near discontinuities. In fact, oscillations at the 156 function discontinuities are visible,

\section{Essential Non-oscillatory Scheme}

${ }_{158}$ Essential Non-oscillatory Schemes (ENO) have been in159 troduced by Harten et al. [1] to solve this problem. The ${ }_{160}$ ENO scheme makes use of adaptive stencils, thus the ${ }_{161}$ use of discontinuity cells is avoided. Let us consider a 162 signal function $f(x)$ with given grid of points of evalu163 ated values such as $v[i]=f\left[x_{i}\right]$.

${ }_{164}$ The ENO scheme reconstructs $f$ from the point values ${ }_{165} v$ assuming that $f$ is piecewise polynomial. This means 166 that for each cell $I_{i} \equiv\left[x_{i-1}, x_{i+1}\right]$ a polynomial inter${ }_{167}$ polant $p_{i}(x)$ is defined using the set of points defined in 168 the stencil $S_{i}$. The idea is to find a stencil of $k+1$ con169 secutive points, including $x_{i-1}$ and $x_{i+1}$, where the signal ${ }_{170} f(x)$ is the smoothest in this stencil when comparing it 171 with the other possible stencils. To evaluate the smooth172 ness of $f(x)$ we can use the Newton divide differences 173 of $f$ :

$$
\begin{gathered}
f\left[x_{0}\right] \equiv f\left(x_{0}\right) ; \\
\quad f\left[x_{0}, x_{1}\right] \equiv \frac{f\left[x_{0}\right]}{\left(x_{0}-x_{1}\right)}+\frac{f\left[x_{1}\right]}{\left(x_{1}-x_{0}\right)} ;
\end{gathered}
$$

175 In general, the $j$-th degree divided difference of $f(x)$ 176 is equivalent to

$$
177 \quad f\left[x_{i-1}, ., x_{i+j-1}\right] \equiv \frac{f\left[x_{i}, ., x_{i+j-1}\right]-f\left[x_{i-1}, ., x_{i+j-2}\right]}{x_{i+j-1}-x_{i-1}} .(2)
$$

178 Starting from a two points stencil

$$
{ }_{179} \quad S_{2}(i)=x_{i-1}, x_{i+1},
$$

180 the linear interpolation of the stencil $S_{2}$ in a Newton 181 form is

$$
182 \quad p_{1}(x)=f\left[x_{i-1}\right]+f\left[x_{i-1}, x_{i+1}\right]\left(x-x_{i-1}\right) .
$$

${ }_{183}$ To expand the stencil we have two possibilities, either ${ }_{184}$ add the left neighbor $x_{i-2}$ or the right one $x_{i+2}$. In both 185 cases this will be a quadratic interpolation polynomial. 186 This will differ from the linear polynomial of eq. 4, by 187 the same function multiplied by two different constants. ${ }_{188}$ These constants are the two 2-nd degrees of divided dif189 ferences of $f(x)$ in two different stencils defined by the 190 left and right neighbors. This procedure is continued un191 til the $k+1$ points in the stencil are reached.

\section{High Order Interpolation Scheme (HOR)}

${ }_{193}$ The typical problem of the ENO scheme is that it ${ }_{194}$ can exhibit oscillatory behavior and is also fairly ex195 pensive in its implementation [22]. As an alterna196 tive, the weighted ENO (WENO) variant has been pro${ }_{197}$ posed $^{1}$. WENO uses a convex combination of all the 198 corresponding interpolating polynomials on the stencil 199 to compute an approximated polynomial for each cell 200 (Figure 3). A convex combination is a linear combina201 tion where the coefficients (weights) are all positive and 202 their sum is equal to 1 . The key points of the reconstruc203 tion scheme are (at $3^{\text {rd }}$ order accuracy):

1. Stencils definition: Taking a cell defined in the interval $\left[x_{i-1 / 2}, x_{i+1 / 2}\right]$ (see Figure 3 ), the stencils are defined as [22]

$$
\begin{aligned}
& S_{1}=\left(x_{i-3 / 2}, x_{i-1 / 2}, x_{i+1 / 2}\right) ; \\
& S_{2}=\left(x_{i-1 / 2}, x_{i+1 / 2}, x_{i+3 / 2}\right)
\end{aligned}
$$

2. Interpolation polynomials: For each stencil the linear interpolation polynomial is computed as

$$
\begin{aligned}
& p_{1}=f\left[x_{i}\right]+\frac{f\left[x_{i}\right]-f\left[x_{i-1}\right]}{\Delta_{x}}\left(x-x_{i}\right) ; \\
& p_{2}=f\left[x_{i}\right]+\frac{f\left[x_{i+1}\right]-f\left[x_{i}\right]}{\Delta_{x}}\left(x-x_{i}\right)
\end{aligned}
$$

where the $f[x]$ elements are the available data points of the function to be reconstructed (red points in Figure 3).

3. Convex combination: The interpolation polynomials are combined following a convex combination

$$
P_{i}=\frac{a_{0}^{i}}{a_{0}^{i}+a_{1}^{i}} p_{1}+\frac{a_{1}^{i}}{a_{0}^{i}+a_{1}^{i}} p_{2}
$$

where

$$
\begin{aligned}
a_{0}^{i} & =\frac{C_{0}^{i}}{\left(\epsilon+(I S)_{1}\right)^{2.0}} ; \\
a_{1}^{i} & =\frac{C_{1}^{i}}{\left(\epsilon+(I S)_{2}\right)^{2.0}}
\end{aligned}
$$

\footnotetext{
${ }^{1}$ WENO schemes have been widely used in computational fluid dynamics; see, for example, Drikakis et al. [23] [24] [25] and references therein
} 


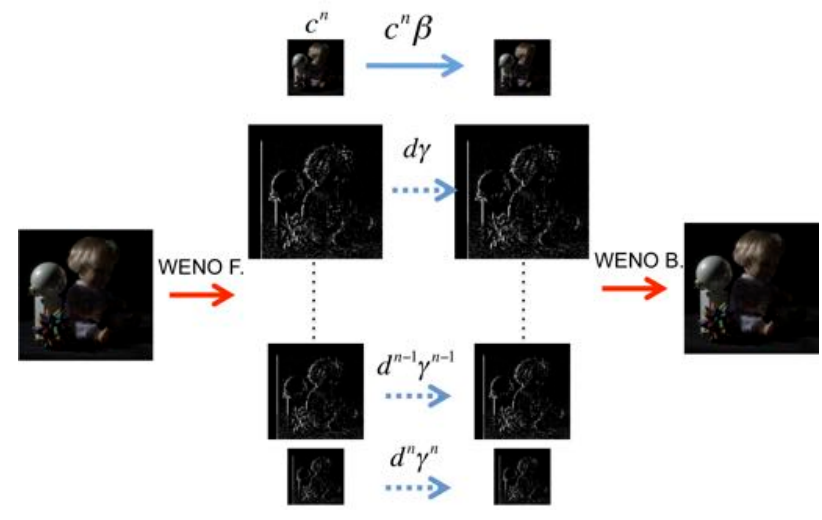

Figure 4: Overview of the present approach. Firstly, a pyramid representation of the input HDR image is produced using a forward wavelet lifting scheme with integrated the HOR interpolation method presented in this paper. Secondly, the coarse level of the pyramid structure (blue continue arrow) and the details levels (blue dashed arrows) are manipulated. Thirdly, the modified pyramid is collapsed to reconstruct the output tone mapped image. This is done, using the backward wavelet lifting scheme with integrated the HOR interpolation model.

\section{${ }_{230}$ 4. HOR Wavelet Scheme}

${ }_{231}$ In this paper we propose a robust smoothing through the 232 use of a polynomial interpolant that makes use of the 233 smoothest stencils. It is integrated in a wavelet scheme ${ }_{234}$ (lifting scheme) to take advantage of the multi-scale ${ }_{235}$ representation such as the capability to retain image in${ }_{236}$ formation at different scale. Figure 4 shows the princi${ }_{237}$ ple of the present approach. Firstly, a pyramid repre${ }_{238}$ sentation of the original input image $I$ is produced us239 ing a forward wavelet lifting WENO scheme. Secondly, 240 the coarse (blue continued arrows) and fine levels (blue ${ }_{241}$ dashed arrow) are manipulated. Thirdly, the modified 242 multiscale representation is collapsed to the output im${ }_{243}$ age using the backwards wavelet lifting WENO scheme. ${ }_{244}$ A multi-scale representation can be obtained by making 245 use of a nested series of decimation $D$ and reconstruc${ }_{246}$ tion $R$ operators. As a $D$ operator, we have used a simple
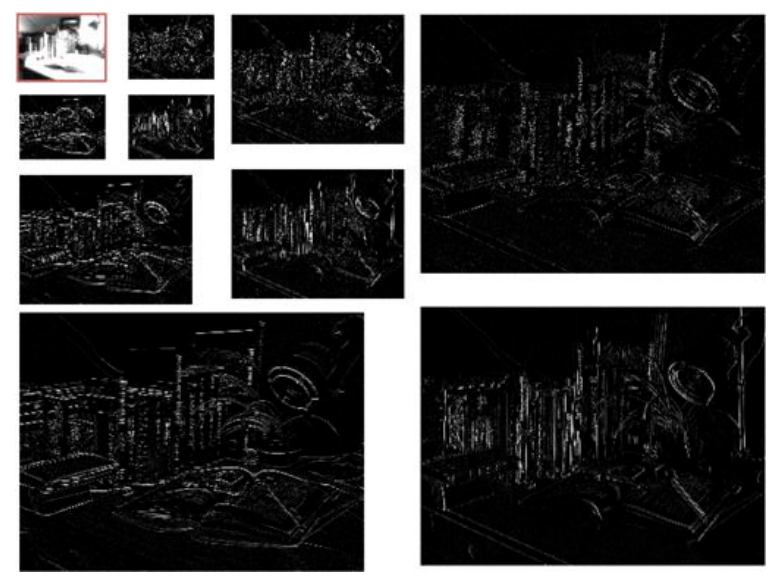

Figure 5: Pyramid image representation, after having applied the forward wavelet lifting WENO scheme. The coarse level is the image at the upper left corner (with red frame). The other images are representing the details, at different levels, for the horizontal, diagonal and vertical directions.

247 splitting operation which separates the pixels of the in248 put at level $k$ in two different grids based on the index 249 number (odd and even). For the $R$ operator the WENO 250 scheme has been employed according to which the level ${ }_{251} k$ is reconstructed from $k+1$ using eq. 9:

$$
\begin{gathered}
\tilde{I}^{k}[x, y]=w_{0}[x, y]\left(I^{k+1}[x-1, y]+\right. \\
\left.\frac{I^{k+1}[x-1, y]-I^{k+1}[x-3, y]}{[x-1, y]-[x-3, y]}([x, y]-[x-1, y])\right) \\
+w_{1}[x, y]\left(I^{k+1}[x-1, y]+\right. \\
\left.\frac{I^{k+1}[x+1, y]-I^{k+1}[x-1, y]}{[x+1, y]-[x-1, y]}([x, y]-[x+1, y])\right) .
\end{gathered}
$$

${ }_{253}$ Eq. 9 is equivalent to eq. 7 where $w_{0}$ and $w_{1}$ are its 254 factorial terms. The difference in the indices between 255 eq. 9 and eq. 7 is due to the fact that we have inserted 256 zero pixels at $k+1$ level and would like to retain in257 teger numbers in the indexing of the grid. Fattal [11] 258 presented a robust average operator, for both type of 259 wavelet approaches, red - black and weighted CDF, 260 making use of an edge stop function to compute the 261 prediction weights. In our case, as described in eq. 7, 262 we present a convex combination of polynomial inter263 polants. However, these polynomial interpolants are lin264 ear, thus we can consider the overall operator as a com265 bination of linear interpolants.

266 At the boundaries of the input image, we have 267 adopted a standard extrapolation approach to generate ${ }_{268}$ the missing values in the stencil. The restored $\tilde{I}^{k}$ level 269 is later used to obtain the details of the $k+1$ level ${ }_{270} d^{k+1}=I^{k}-\tilde{I}^{k}$. To preserve the overall sum of the coarse ${ }_{271}$ elements $I_{k+1}$, and based on the fact that the operator $R$ 272 can be seen as combination of two linear interpolants 
273 we have decided to use a linear interpolator as update274 operator $U$ :

$275 \quad U\left(d^{k+1}\right)[x, y]=\frac{d^{k+1}[x-1, y]+d^{k+1}[x+1, y]}{4} ;(10)$

276 and the level $k+1$ of coarse elements is updated using ${ }_{277} I^{k+1}=I^{k+1}+U\left(d^{k+1}\right)$.

278 This process is repeated both for the rows and 279 columns of the input image.

280 Discussion An example of the behaviour of the 281 present HOR, integrated in the wavelet scheme, is 282 shown in Figure 5. The coarse, $c$, and 'details' coeffi283 cients, $d$, (vertical, diagonal and horizontal) for three ${ }_{284}$ levels are shown. Edges are detected by the wavelet 285 scheme avoiding the influence of pixels on both sides 286 at each scale. This is obtained without the introduction 287 of an edge stop function utilized for the computation of 288 the set of weights used in the interpolation step as pro289 posed by Fattal [11].

\section{5. Tone Mapping Manipulation}

291 In this subsection, we will show how to make use 292 of the proposed technique in the classical tone ma293 nipulation problem. Tone manipulation allows to re294 duce the intensity of the luminance range of HDR con295 tent. This objective is achieved through compression of 296 large-scale variation and keeping the fine level informa297 tion. The filtering approach is applied to the natural log298 arithmic scale of the luminance, keeping the color ratio 299 unaltered as in Paris et al. [9], using a gamma correction 300 of 2.2 .

301 To manipulate the tone and the details of the input HDR 302 image, we have followed a similar approach to the one 303 used by Fattal [11]. The tone is linearly manipulated 304 modifying the coarse coefficient $c$ of the coarsest level $n$ 305 through a parameter $\beta$, as $\beta c^{n}$. This allows us to achieve 306 the compression of the vast dynamic range available in 307 the input HDR image. A second parameter $\gamma$ is used 308 to manipulate the details. This is obtained from the 309 progressive decreasing of the 'details' coefficients $d^{k}$, ${ }_{310}$ such as $\gamma^{k} d^{k}$ where $k$ is the number of levels varying be311 tween 1 to $n$. The $\beta$ and $\gamma$ parameters are in the range of $312(0.0,1.0]$.

${ }_{313}$ Since our approach shares several aspects with the tech${ }_{314}$ nique presented by Fattal [11], we first provide an anal315 ysis and comparison to show how the present technique 316 performs with respect to the preservation of edges, 317 while at the same time adjusting the tone of the input 318 image.

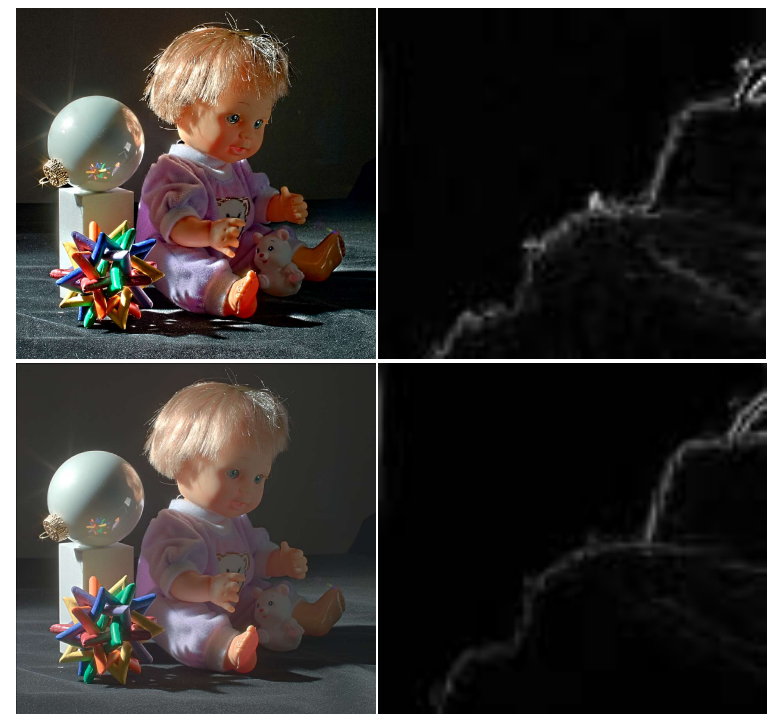

Figure 6: Comparisons with state-of-the-art method Fattal's method [11] . $1^{\text {st }}$ row: Fattal [11] using wavelet Red and Black model with $\alpha=0.8, \beta=0.11$ and $\gamma=0.68-2^{\text {nd }}$ row: the present approach with $\beta=0.3$ and $\gamma=0.7-2^{\text {nd }}$ column: Gradient of a zoomed area, it showing the degree of edge preservation.
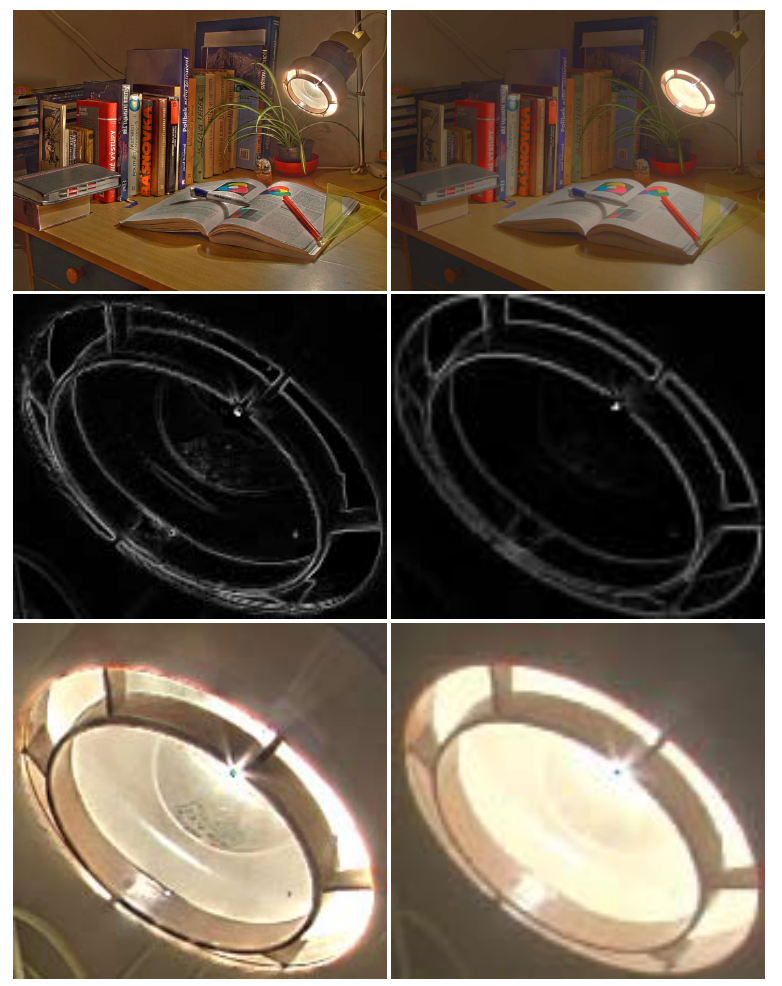

Figure 7: Comparisons of different methods. $1^{\text {st }}$ column: Fattal [11] using wavelet Red and Black model with parameters as per web project page [26] - $2^{\text {nd }}$ column: The present approach with $\beta=0.7$ and $\gamma=0.9-2^{\text {nd }}$ row: Gradient of the zoomed area in the $3^{\text {rd }}$ row. Distortions at the edges are visible. 

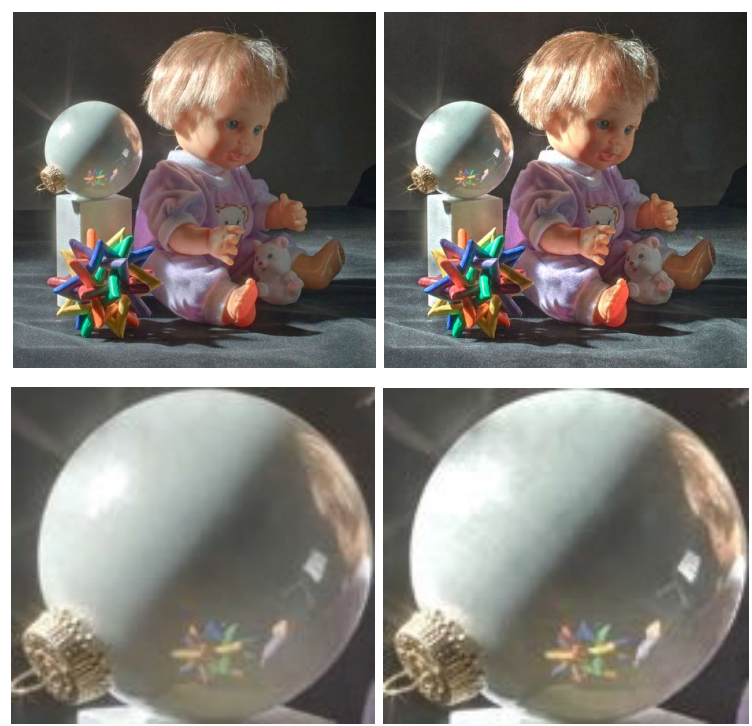

(a) Fattal $[11] \beta=0.7$ - Weak

(b) Fattal $[11] \beta=0.5$ - Strong

Figure 8: Results from the application of Fattal's et al. [11] technique making use of the new contractive concave mapping as specified in [26].

319 The present technique produces results comparable 320 to this state-of-the-art operator, while offering the ad321 vantage of not using an extra edge-stop function. The 322 technique of [11] is capable to capture more details but ${ }_{323}$ at the cost of introducing some distortions at the edge ${ }_{324}$ level, as shown in Figures 7 (a) (zoomed lamp area and 325 its edge map) and 6 (b) (edge map).

${ }_{326}$ One may reduce these distortions by making use of a 327 new compression technique, as suggested in [26] (Fig328 ure 8 ). However, artifacts may appear as shown in Fig329 ure 8 (b) $2^{\text {nd }}$ row.

\section{${ }_{330}$ 6. Experimental Results}

${ }_{331}$ The HOR approach has been implemented in Matlab ${ }_{332}$ and the experiments have been performed on a Mack$3 з 3$ book air with Intel i7-core CPU $1.8 \mathrm{GHz}, 64$-bit ma${ }_{334}$ chine and 4GB of RAM. We have compared our tech${ }_{335}$ nique with the latest edge aware state-of-the-art multi$3 з 6$ scale approaches, applied to the tone mapping problem, ${ }_{337}$ such as[11, 9, 10]. We have used the Matlab code as ${ }_{338}$ well as parameters provided by the authors.

3з9 We have chosen the set of images shown in Figure 9. ${ }_{340}$ This set consists of 18 images with different dynamic ${ }_{341}$ range that span from outdoor to indoor and from light to 342 dark illumination conditions.

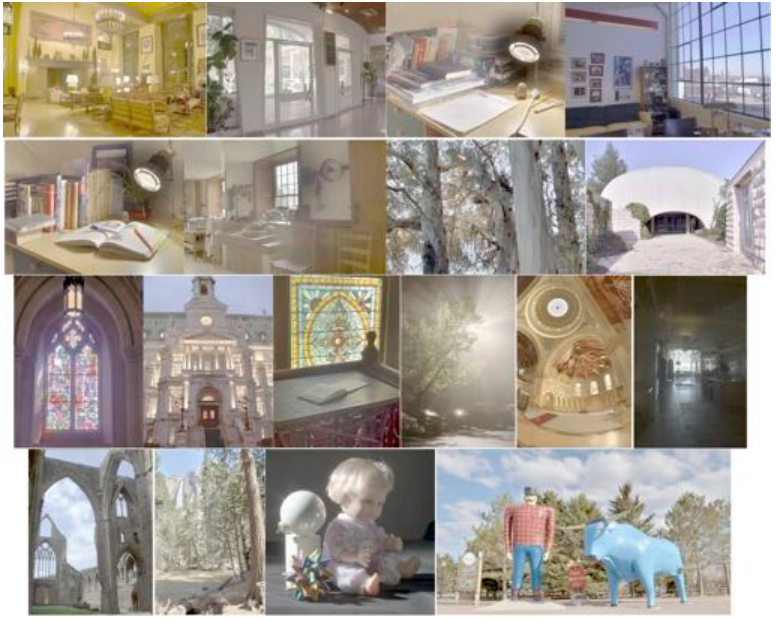

Figure 9: Images used in the experiments. The numbering in Tables 1 and 2 follows the order of the images from the top to the bottom and from the left to the right.

\section{${ }_{343}$ 6.1. Quality}

${ }_{344}$ To provide a fair comparison, we have selected the 345 parameters of the different techniques to convey sim${ }_{346}$ ilar appearance in term of contrast, edges and details 347 preservation to all the techniques presented in this com348 parison.

${ }_{349}$ We may observe that the DRIM metric is measur350 ing changes in contrast, in other words the overall ap351 pearance of the image, and it is not able to detect if 352 small-scale details are not well preserved. On the other ${ }_{353}$ hand, edge-aware techniques are able to preserve well 354 small-scale details. This is preserved intrinsically by the 355 mechanisms described in the previous sections as well 356 as by the results shown here that are comparable with 357 the existing state-of-the-art edge aware technique [11].

358 Based on the fact that small-scale details are to certain ${ }_{359}$ extent well reproduced by the edge-aware techniques, ${ }_{360}$ our objective was to examine how these techniques are 361 able to convey the overall appearance of the input HDR 362 into the tone mapped result. In doing so, we have de363 cided to use the DRIM metric as specified below.

${ }_{364}$ Since the DRIM metric accepts $c d / m^{2}$ values, the in${ }_{365}$ put images need to be calibrated. In the case of the tone 366 mapped input image, we need to linearize the input sig367 nal and then map it to the dynamic range of the display 368 where the image will be visualized. In our case, the $\gamma$ ${ }_{369}$ value used for the linearization step is 2.2 , and the dy370 namic range chosen is $[0.5,100] \mathrm{cd} / \mathrm{m}^{2}$. In the case 371 of the HDR input image, there was no need to linearize 372 the signal, and the dynamic range has been chosen as ${ }_{373}[0.015,3000] \mathrm{cd} / \mathrm{m}^{2}$.

\section{$374 \quad$ DRIM Results Discussion}



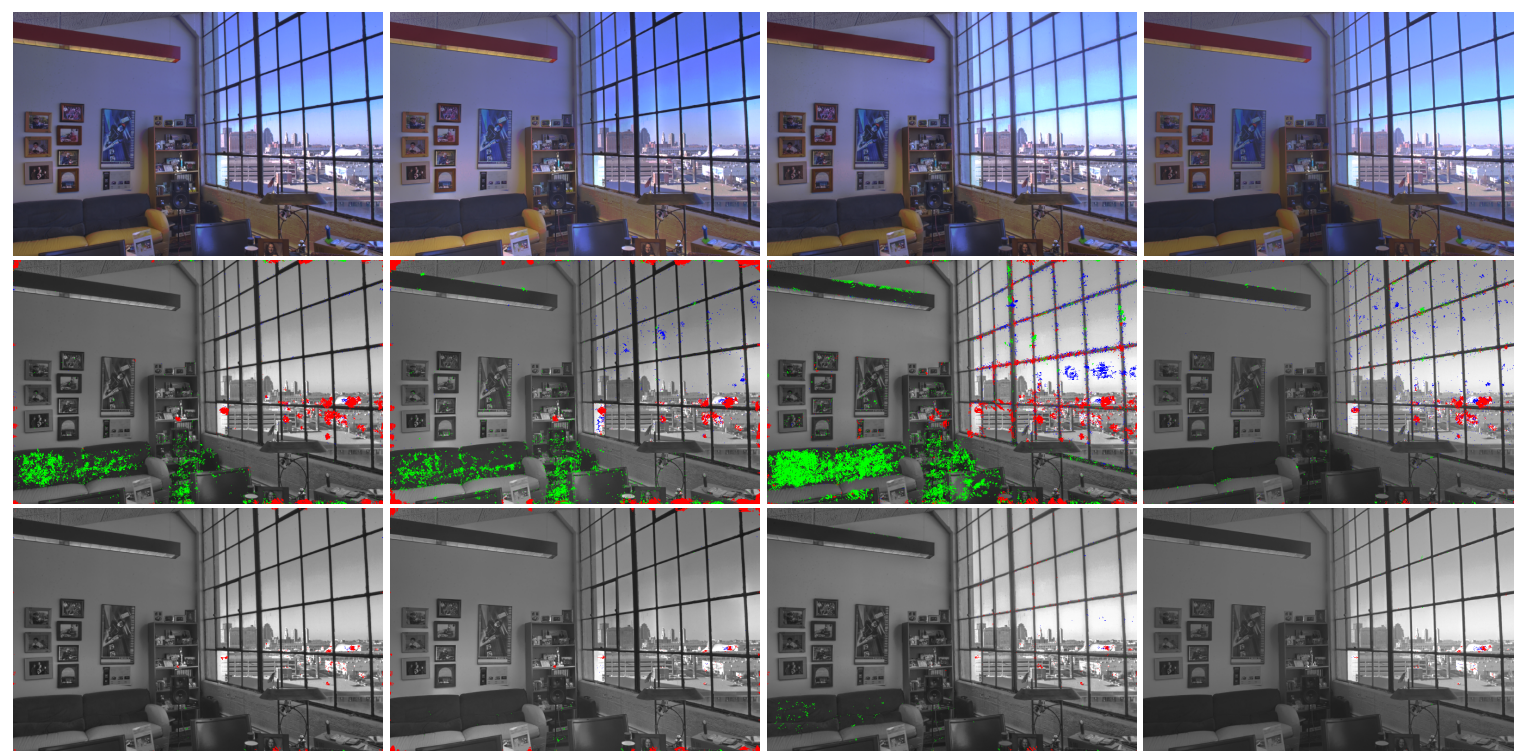

Figure 10: Output and DRIM comparison with state-of-the-art edge aware approaches. $1^{\text {st }}$ - row output of the edge aware technique; $2^{\text {nd }}$ row DRIM metric [12] with probability of 75\%; $3^{\text {rd }}$ row - DRIM metric [12] with probability of $95 \%$. Parameters used - Farbmann et al. [10] multiscale approach balanced - Fattal's [11] $\alpha=0.9, \beta=0.19$ and $\gamma=0.5$ - Paris et al. [9] $\sigma_{r}=\log (2.5), \alpha=0.5$ and $\beta=0.0$ (for conveying the local effect) The Present HOR $\beta=0.7, \gamma=0.9$.

375 Tables 1 and 2 show the results of the DRIM metric 376 applied to the test set images. The numbers represent 377 the percentage of pixels with probability for the distor378 tion to be perceived by the HVS. Tables 1 and 2 show ${ }_{379}$ the results with probability $95 \%$ and $75 \%$, respectively. ${ }_{380}$ The colors used to depict the type of distortion are the 381 same with those used to describe the distortion - $R$ (red) 382 reversal, - $G$ (green) lost and - $B$ (blue) amplification of ${ }_{383}$ contrast. We have colored the methods that show the ${ }_{384}$ higher probability, as well as the ones that show signifi385 cant percentage of pixels with the specified probability. ${ }_{386}$ In the case of probability $95 \%$, the significant distortion 387 introduced by the state-of-the-art edge aware methods, 388 as well as by the present HOR is mostly due to the loss 389 of contrast; neither reversal nor amplification of con390 trast are significant. The lost of contrast is attributed to 391 the fact that the edge-aware methods are using simple 392 linear scaling for compressing the large luminance dy393 namic range. This may affect the overall preservation of ${ }_{394}$ local contrast. With probability $95 \%$ the state-of-the-art 395 methods may present high percentage of pixels affected ${ }_{396}$ by loss of contrast. This is the case of the images 1,3 , $3975,11,13$ and 14. In most of the other cases, this number 398 is negligible. For the images 1, 13 and 14, the HOR ${ }_{399}$ shows a slightly higher percentage value for the loss 400 of contrast. However, this value is either comparable 401 or lower than the value provided by the state-of-the-art 402 edge-aware methods. We have also tried to analyze the
${ }_{403}$ results of the DRIM metric at lower probability such 404 as $75 \%$ and the results are shown in Table 2. As ex405 pected, the percentage of pixels is drastically increased 406 and more images are affected by a significant percent407 age value. In this case, reversal of contrast (red) and in 408 some cases amplification of contrast (blue) may appear. 409 In the case of loss of contrast the Fattal [11] and Paris et 410 al. [9] results show that the majority of the images are 411 affected by this type of distortion. This type of distor412 tion also affects the present HOR, but when compared ${ }_{413}$ with the state-of-the-art edge-aware methods shows a ${ }_{414}$ lower percentage of pixels affected by this distortion.

415 Only in the case of image 18 the present HOR shows ${ }_{416}$ higher value for the loss of contrast. However, this per417 centage value is quite small and it is not actually per418 ceivable by the HVS. The results are also affected by ${ }_{419}$ the reversal of contrast. In particular, several results 420 of Fattals [11] method are showing this distortion. The ${ }_{421}$ present HOR shows reversal of contrast higher than the 422 other methods only for three images (11, 12 and 18). ${ }_{423}$ Finally, the amplification of contrast (blue) does almost 424 not exist, and the only image that is affected by using 425 the proposed HOR is the image 15.

\section{DRIM Visual Analysis}

${ }_{427}$ Figures 10, 11, 13 and 14 show results with the 428 corresponding DRIM distortion maps. Figure 10 and ${ }_{429}$ Figure 11 compares the DRIM maps at probability $75 \%$ ${ }_{430}\left(2^{\text {nd }}\right.$ row) and at $95 \%$ ( $3^{\text {rd }}$ row) for each output result. 


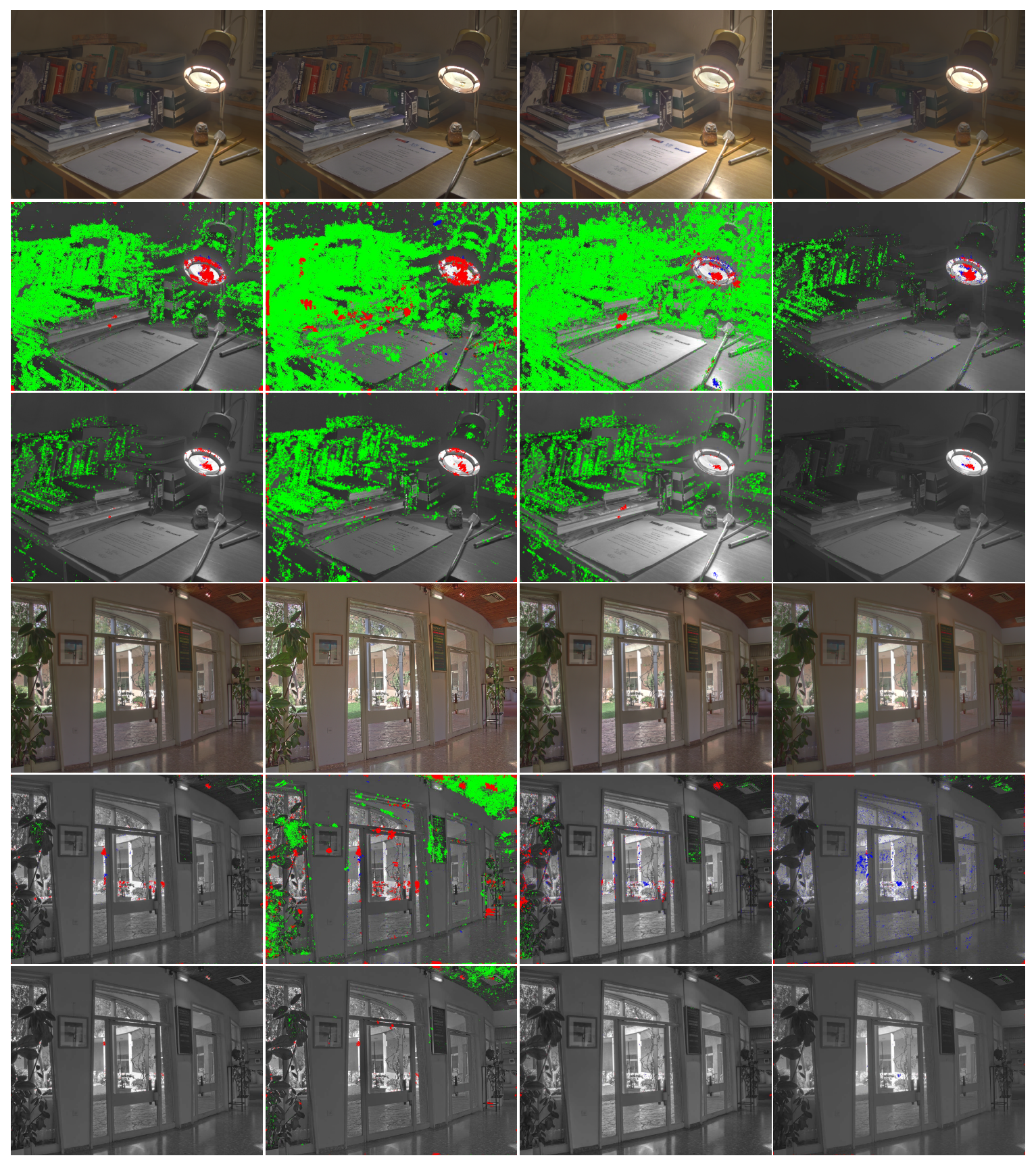

Figure 11: Output and DRIM comparison with state-of-the-art edge aware approaches. $1^{\text {st }}$ - row output of the edge aware technique; $2^{\text {nd }}$ row DRIM metric [12] with probability of $75 \% ; 3^{\text {rd }}$ row - DRIM metric [12] with probability of $95 \%$ (for both images). Parameters used - Farbmann et al. [10] multiscale approach balanced - Fattal's [11] $\alpha=0.8, \beta=0.12$ and $\gamma=0.9$ - Paris et al. [9] $\sigma_{r}=\log (2.5), \alpha=0.5$ and $\beta=0.0$ (for conveying the local effect) - The Present HOR $\beta=0.7, \gamma=0.9$. 


\begin{tabular}{|c|c|c|c|c|}
\hline Image & Farbman [10] & Fattal [11]. & Paris [9] & HOR \\
\hline 1 AhwahneeGL & R 0.28 G 3.72 B 0.0 & R 0.22 G 3.0 B 0.0 & R 0.6 G 6.16 B 0.0 & R 0.26 G 5.24 B 0.0 \\
\hline 2 Belgium & $\mathrm{R} 0.0 \mathrm{G} 0.0 \mathrm{~B} 0.0$ & R $0.11 \mathrm{G} 0.35$ B 0.0 & R 0.0 G 0.0 B 0.0 & R 0.0 G 0.0 B 0.0 \\
\hline 3 Cadik1 & R 0.0 G 1.34 B 0.0 & R 0.2 G 5.1 B 0.73 & R 0.0 G 3.44 B 0.0 & R 0.0 G 0.0 B 0.0 \\
\hline 4 smallOffice & $\mathrm{R} 0.0 \mathrm{G} 0.0 \mathrm{~B} 0.0$ & R 0.14 G 0.0 B 0.0 & R 0.0 G 0.0 B 0.0 & R 0.0 G 0.0 B 0.0 \\
\hline 5 Cadik2 & $\mathrm{R} 0.0 \mathrm{G} 4.0 \mathrm{~B} 0.0$ & R $0.0 \mathrm{G} 3.02$ B 0.0 & $\mathrm{R} 0.0 \mathrm{G}$ & R 0.0 G 0.89 B 0.0 \\
\hline 6 Kitchen & R 0.0 G 0.0 B 0.0 & R 0.19 G 0.22 B 0.0 & R 0.0 G 0.8 B 0.0 & R 0.0 G 0.0 B 0.0 \\
\hline 7 GroveD & $\mathrm{R} 0.0 \mathrm{G} 0.0 \mathrm{~B} 0.0$ & R 0.29 G 0.0 B 0.0 & R 0.0 G 0.0 B 0.0 & R 0.0 G 0.0 B 0.0 \\
\hline 8 Synagouge & R 0.0 & R 0.13 & $\mathrm{R} 0.0 \mathrm{G}$ & R 0.0 G 0.0 B 0.0 \\
\hline 9 Cathedral & R 0.0 & $\mathrm{R} 0.23$ & $\mathrm{R} 0.0 \mathrm{G}$ & R 0.17 G 0.0 B 0.0 \\
\hline 10 Clockbui & R 0.0 & $\mathrm{R} 0.14 \mathrm{G}$ & R 0.0 G 0.0 B 0.0 & R 0.0 G 0.0 B 0.0 \\
\hline 11 Desk & R 0.0 G 0.86 B 0.0 & $\mathrm{R} 0.31 \mathrm{G} 3.0 \mathrm{~B} 0.0$ & R 0.11 G 1.78 B 0.0 & R 0.23 G 0.96 B 0.0 \\
\hline 12 FogMap & R 0.13 G 0.8 B 0.0 & R 0.14 G 0.79 B 0.0 & R 0.0 G 0.0 B 0.0 & R 0.25 G 0.74 B 0.0 \\
\hline 13 Memorial & R $0.1 \mathrm{G} 2.4 \mathrm{~B} 0.0$ & R 0.27 G 7.1 B 0.0 & R 0.24 G 7.9 B 0.0 & R 0.13 G 3.9 B 0.0 \\
\hline 14 DesignCenter & 4.35 B 0.0 & 6 B 0.0 & R 0.0 G 18.0 B 0.0 & R 0.18 G 4.2 B 0.0 \\
\hline 15 Tinterna & R 0.0 G 0.0 B 0.0 & R 0.27 G 0.0 B 0.0 & R 0.0 G 0.0 B 0.0 & R 0.0 G 0.0 B 0.0 \\
\hline 16 Yosemite & $\mathrm{R} 0.0 \mathrm{G} 0.0 \mathrm{~B} 0.0$ & $\mathrm{R} 0.32 \mathrm{G} 0.0$ B 0.0 & R 0.0 G 0.0 B 0.0 & R 0.0 G 0.0 B 0.0 \\
\hline 17 Doll & $\mathrm{R} 0.0 \mathrm{G} 0.0 \mathrm{~B} 0.0$ & $\mathrm{R} 0.0 \mathrm{G} 0.0 \mathrm{~B} 0.0$ & $\mathrm{R} 0.0 \mathrm{G} 0.0 \mathrm{~B} 0.0$ & R 0.31 G 0.13 B 0.0 \\
\hline 18 Paull & $\mathrm{R} 0.0 \mathrm{G} 0.0 \mathrm{~B} 0.0$ & $\mathrm{R} 0.0 \mathrm{G} 0.0 \mathrm{~B} 0.0$ & $\mathrm{R} 0.0 \mathrm{G} 0.0 \mathrm{~B} 0.0$ & R 0.11 G 0.0 B 0.0 \\
\hline AVERAGE & R 0.028 G 0.97 B 0.0 & R 0.19 G 2.38 B 0.041 & R 0.053 G 2.2 B 0.0 & R 0.09 G 0.89 B 0.0 \\
\hline
\end{tabular}

Table 1: DRIM results over the set of images presented in Figure 9. We show the percentage of pixels with probability of $95 \%$ that present the distortion of reverse $(R)$, loss $(G)$, or amplification $(B)$ of contrast.

431 The visual analysis of the results shows that in the ${ }_{432}$ case of probability $75 \%$ the state-of-the-art methods ${ }_{433}$ show a consistent number of distorted pixels localized ${ }_{434}$ in large areas, when compared with the present HOR. ${ }_{435}$ On the other hand, when the probability increases to ${ }_{436} 95 \%$, the size of these areas are either reduced or are ${ }_{437}$ almost not affected by any distortion. However, in some 438 cases the state-of-the-art methods are still showing large 439 areas of lost of contrast (green) and reversal of contrast 440 (red).

$441 \quad$ Figures 13 and 14 are showing other results with the 442 distortion maps with probability at $95 \%$, where the all ${ }_{443}$ methods are showing similar behavior..

\section{Comparison with Simpler TMO's}

445 One can observe that the global operators are faster 446 and convey an overall better appearance (Artusi et ${ }_{447}$ al. [28]). For this purpose, we have computed the ${ }_{448}$ DRIM maps for a well known global version of two ${ }_{449}$ TMOs published by by Reinhard et al. [27] and Drago 450 et al. [27]. The comparison is limited to the global op451 erator showing that the quality of the results is not com${ }_{452}$ parable with the state-of-the-art edge aware techniques. 453 The results are shown in Figure 12 for the distortion ${ }_{454}$ maps at probability of $95 \%$.

${ }_{455}$ The results reveal that the DRIM obtained for the ${ }_{456}$ Reinhard et al. [27] and the Drago et al. [27] opera457 tors often show larger areas of amplification of contrast; 458 see the window area in Figure 12, in comparison with ${ }_{459}$ the results obtained by the majority of the edge-aware
460 techniques employed in this experiment.

461 Figure $12\left(2^{n} d\right.$ row shows reversal of contrast, in 462 large areas of the window, for both global operators. ${ }_{463}$ On the other hand, the edge-aware techniques have very ${ }_{464}$ tiny areas affected by reversal of contrast. Moreover, 465 we emphasize in general that global operators are not ${ }_{466}$ designed for edge-awareness and do not encapsulate 467 mechanisms for retaining the fine details at different ${ }_{468}$ spatial scale, as in the case of the present HOR and 469 edge-aware techniques.

\section{6.2. Computational Analysis}

471 Another aspect that needs to be taken into account 472 is the computational cost associated with the different ${ }_{473}$ algorithms. Here, we have performed a computational 474 cost analysis for the proposed technique versus other 475 state-of-the-art techniques.

476 Our approach presents computational complexity and ${ }_{477}$ associated cost comparable to the one presented in [11, 478 10] and outperforming the method of [9] .

479 Specifically, the method presented by Paris et al. [9] 480 requires $1738 \mathrm{sec}$ to process an image size of $800 \times 525$, ${ }_{481} 420 \mathrm{sec}$ for an image size of 400x262 and $190 \mathrm{sec}$. for 482 an image size of $267 \times 174$. When compared with the 483 computational cost of our method and the approaches ${ }_{484}$ of $[11,10]$, the computational cost is significantly re485 duced: 14 sec to process an image size of $800 \times 525,3$ $486 \mathrm{sec}$ for an image size of 400x262, and $1 \mathrm{sec}$ for an im487 age size of $267 \times 174$. 


\begin{tabular}{|c|c|c|c|c|}
\hline Image & Farbman [10] & Fattal [11]. & Paris [9] & HOR \\
\hline 1 AhwahneeGL & R 1.0 G 15.0 B 0.17 & R 0.91 G 12.6 B 0.15 & R 1.96 G 21 B 0.2 & R 1.33 G 18.5 B 0.11 \\
\hline 2 Belgium & R 0.13 G 0.0 B 0.0 & R 0.9 G 2.73 B 0.0 & R 0.27 G 0.18 B 0.15 & R 0.14 G 0.0 B 0.4 \\
\hline 3 Cadik1 & R 0.4 G 9.2 B 0.0 & R $1.1 \mathrm{G} 16.6$ B 0.0 & R 0.31 G 17.4 B 0.12 & R 0.13 G 1.72 B 0.14 \\
\hline 4 smallOffice & R 0.43 G 0.58 B 0.0 & R 0.57 G 0.67 B 1.63 & R 0.78 G 1.77 B 0.45 & R 0.24 G 0.0 B 0.24 \\
\hline 5 Cadik2 & R 0.4 G 16.7 B 0.0 & R 0.44 G 12.8 B 0.0 & R 0.45 G 25 B 0.18 & R 0.12 G 6.7 B 0.0 \\
\hline 6 Kitchen & R $0.0 \mathrm{G} 0.0 \mathrm{~B} 0.0$ & R 0.92 G 4.9 B 0.0 & R 0.3 G 0.11 B 0.16 & R 0.0 G 0.59 B 0.0 \\
\hline 7 GroveD & R 0.15 G 0.0 B 0.13 & R 5.9 G 0.42 B 0.67 & R 0.5 G 0.0 B 0.32 & R 0.79 G 0.0 B 0.4 \\
\hline 8 Synagouge & R 0.18 G 0.1 B 0.15 & R 2.4 G 3.8 B 0.1 & $\mathrm{R} 0.1 \mathrm{G} 0.0 \mathrm{~B} 0.3$ & R 0.3 G 0.38 B 0.81 \\
\hline 9 Cathedral & R 0.0 G 0.0 B 0.15 & R 2.29 G 1.14 B 0.57 & R 0.64 G 0.0 B 0.0 & R 1.66 G 0.76 B 2.0 \\
\hline 10 Clockbui & R 0.29 G 0.0 B 0.0 & R 0.61 G 0.0 B 0.20 & R 1.12 G 0.0 B 0.6 & R 0.6 G 0.18 B 0.24 \\
\hline 11 Desk & R 0.4 G 3.9 B 0.12 & R 2.28 G 10.2 B 0.18 & R 1.9 G 8.6 B 0.54 & R 3.03 G 5.11 B 0.73 \\
\hline 12 FogMap & R 0.34 G 0.73 B 0.0 & R 0.0 G 2.15 B 0.03 & R $1.1 \mathrm{G} 19$ B 0.0 & $\mathrm{R} 2.45 \mathrm{G} 18.6 \mathrm{~B} 0.0$ \\
\hline 13 Memorial & R 0.6 G 14.8 B 0.0 & $\mathrm{R} 1.55 \mathrm{G} 23.5 \mathrm{~B} 0.0$ & R 1.9 G 26.9 B 0.0 & R 0.97 G 18.7 B 0.0 \\
\hline 14 DesignCenter & R $0.31 \mathrm{G} 21.7$ B 0.0 & R $3.8 \mathrm{G} 30.5$ B 0.0 & R 0.55 G 28.7 B 0.0 & $\mathrm{R} 1.35 \mathrm{G} 22.0 \mathrm{~B} 0.0$ \\
\hline 15 Tinterna & R 0.5 G 0.0 B 0.6 & R 3.7 G 0.63 B 0.55 & R 0.18 G 0.11 B 0.57 & R 0.17 G 0.0 B 4.36 \\
\hline 16 Yosemite & $\mathrm{R} 0.23 \mathrm{G} 0.0 \mathrm{~B} 0.0$ & R 4.1 G 0.49 B 0.21 & R 0.38 G 0.0 B 0.48 & R 0.15 G 0.0 B 0.45 \\
\hline 17 Doll & R 0.13 G 0.0 B 0.35 & R 0.82 G 3.6 B 0.14 & R 0.35 G 0.31 B 0.2 & R 1.7 G 1.5 B 0.53 \\
\hline 18 Paull & R 0.18 G 0.0 B 0.1 & R 1.8 G 0.36 B 0.25 & R 0.24 G 0.1 B 0.4 & R 2.5 G 1.22 B 0.4 \\
\hline AVERAGE & R 0.32 G 4.81 B 0.098 & R 1.89 G 7.06 B 0.26 & R 0.72 G 8.29 B 0.26 & R 0.98 G 5.33 B 0.59 \\
\hline
\end{tabular}

Table 2: DRIM results over the set of images presented in Figure 9. We show the percentage of pixels with probability of $75 \%$ that present the distortion of reverse $(R)$, loss $(G)$, or amplification $(B)$ of contrast.

488 Recently, Aubry et al. [14] presented a fast im489 plementation of Paris et al. [9] technique that signif490 icantly improves its computational performances (50 491 times faster). However, our comparison is done on the 492 Matlab implementation of the all techniques used in the ${ }_{493}$ evaluation, as provided by the authors, without includ494 ing any optimization. Even if we apply the 50-fold im495 provement in the measured time of the Matlab imple496 mentation of Paris et al. [9], the present HOR delivers 497 an excellent overall performance.

\section{7. Concluding remarks}

499 We have introduced a new edge preserving tech500 nique that makes use of a HOR method, which is able 501 to preserve edges without introducing artifacts and re502 ducing any changes in the image structure when com${ }_{503}$ pared to the state-of-the-art edge preserving operators. ${ }_{504}$ The present method does not require an extra stop-edge 505 function, thus offering simplicity. Futhermore, its com506 putational cost increases linearly in time. We have 507 demonstrated the accuracy of the present technique on a 508 variety of images and parameter settings. The use of the 509 HOR technique in other applications such as details en510 hancement and image colorisation is also possible and 511 will be part of future work. The proposed HOR tech512 nique will be further implemented in graphics hardware 513 with reference to video applications, allowing substan514 tial improvements in computational performance.

\section{8. Acknowledgments}

${ }_{516}$ This work was partially supported by Ministry of ${ }_{517}$ Science and Innovation Subprogramme Ramon y Cajal ${ }_{518}$ RYC-2011-09372, TIN2013-47276-C6-1-R from Span519 ish government, 2014 SGR 1232 from Catalan gov${ }_{520}$ ernment, NSFC-Guangdong Joint Fund (U1135003, ${ }_{521}$ Natural Science Foundation of China (NSFC) (No. $\left.{ }_{522} 61320106008\right)$.

\section{${ }_{523}$ References}

524 [1] A. Harten, B. Engquist, S. Osher, S. Chakravarthy, Uniformly high order essentially non-oscillatory schemes, Journal of Computational Physics 1987.

[2] C. W. Shu, Essentially non-oscillatory and weighted essentially non-oscillatory schemes for hyperbolic conservation laws, Tech. rep., ICASE Report No. 97-65, NASA - CR-97-206253 (1997).

[3] T. F. Chan, H. M. Zhou, Eno-wavelet transforms for piecewise smooth functions, SIAM Journal on Numerical Analysis 40 (2002) 1369-1404.

[4] H. Boucenna, M. Halimi, Image segmentation by the level set methods using third order weno, 5th International Conference: Sciences of Electronic, Technologies of Information and Telecommunications 2009.

[5] P. Perona, J. Malik, Scale-space and edge detection using anisotropic diffusion, IEEE Transactions on Pattern Analysis and Machine Intelligence 12 (1990) 629-639.

[6] C. Tomasi, R. Manduchi, Bilateral filtering for gray and color images, Proceedings of the IEEE International Conference on Computer Vision (1998) 839-846.

[7] Z. Su, X. Luo, A. Alessandro, A novel image decomposition approach and its applications., The Visual Computer 29 (10) (2013) 1011-1023. 

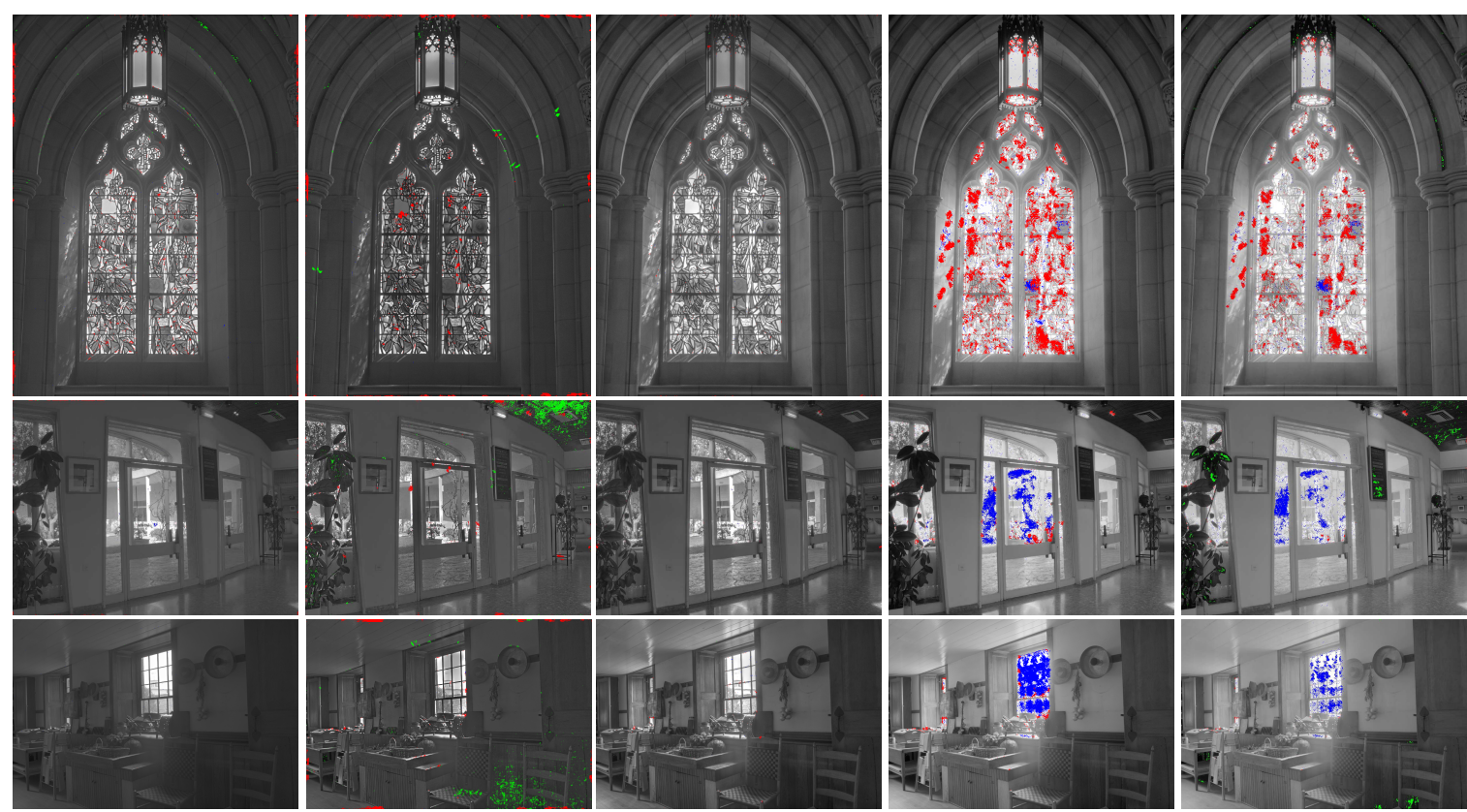

Figure 12: DRIM comparison with state-of-the-art edge aware approaches and simple TMO. DRIM metric [12] with probability of 95\%. Farbman et al. [10] DRIM output is omitted because of the similarities in the results with the one obtained with the present HOR.

B. C. A. Buades, J. M. Morel, The staricasing effect in neighborhood filters and its solution, IEEE Journal on Transactions on Image Processing 15 (2006) 1499-1505.

] P. Silvan, S. W. Hasinoff, J. Kautz, Local laplacian filters: Edge aware image processing with a laplacian pyramid, ACM Transactions on Graphics (Proceedings of ACM SIGGRAPH 2011).

0] Z. Farbman, R. Fattal, D. Lischinski, R. Szeliski, Edgepreserving decompositions for multi-scale tone and detail manipulation, in: ACM SIGGRAPH 2008 Papers, SIGGRAPH '08, ACM, 2008, pp. 67:1-67:10.

1] R. Fattal, Edge-avoiding wavelets and their applications, ACM Trans. Graph. 28 (3) (2009) 1-10.

2] T. O. Aydin, R. Mantiuk, K. Myszkowski, H.-P. Seidel, Dynamic range independent image quality assessment, ACM Trans. Graph. 27 (3) (2008) 69:1-69:10.

3] F. Banterle, A. Artusi, K. Debattista, A. Chalmers, Advanced High Dynamic Range Imaging: Theory and Practice, AK Peters (CRC Press), Natick, MA, USA, 2011.

4] M. Aubry, S. Paris, S. Hasinoff, J. Kautz, F. Durand, Fast and robust pyramid-based image processing, Tech. rep. (2011).

R. Fattal, D. Lischinski, M. Werman, Gradient domain high dynamic range compression, ACM Trans. Graph. 21 (3) (2002) 249-256.

] J. Chen, S. Paris, F. Durand, Real-time edge-aware image processing with the bilateral grid, ACM Transactions on Graphics (Proceedings of ACM SIGGRAPH 2007)

7] J. Tumblin, G. Turk, Lcis: A boundary hierarchy for detailpreserving contrast reduction, ACM Transactions on Graphics (Proceedings of ACM SIGGRAPH 1999).

P. J. Burt, Edward, E. H. Adelson, The laplacian pyramid as compact image code, IEEE Transactions on Communications 31 (1983) 532-540.

Graphics and Image Processing 16 (1) (1981) 20 - 51

[20] A. N. Akansu, P. R. Haddad, Multiresolution Signal Decompo-
581 sition: Transforms, Subbands, and Wavelets, Academic Press, $582 \quad 1992$ and 2000 (2nd Ed.)

583 [21] Y. Li, L. Sharan, E. H. Adelson, Compressing and companding 584 high dynamic range images with subband architectures, ACM 585 Trans. Graph. 24 (2005) 836-844.

86 [22] X. Liu, S. Osher, T. Chan, Weighted essentially non-oscillatory 587 schemes, Journal of Computational Physics 115 (1994) 200212.

589 [23] A. Mosedale, D. Drikakis, Assessment of very high-order of accuracy in les models, ASME Journal of Fluids Engineering 129 (2007) 1497-1503.

592 [24] B. Thornber, A. Mosedale, D. Drikakis, On the implicit large eddy simulations of homogeneous decaying turbulence, Journal of Computational Physics 226 (2007) 1902-1929.

5 [25] D. Drikakis, M. Hahn, A. Mosedale, T. B., Large eddy simulation using high resolution and high order methods, Philosophical Transactions Royal Society A 367 (2009) 2985-2997.

[26] R. Fattal[link] URL http://www.cs.huji.ac.il/ raananf/projects/eaw/

600 [27] E. Reinhard, M. Stark, P. Shirley, J. Ferwerda, Photographic tone reproduction for digital images, ACM Trans. Graph. 21 (3) (2002) 267-276

3 [28] A. Artusi, O. Akyz, Ahmet, B. Roch, D. Michael, Y. Chrysanthou, A. Chalmers, Selective local tone mapping, proceedings IEEE International Conference on Image Processing (ICIP) 2013. 


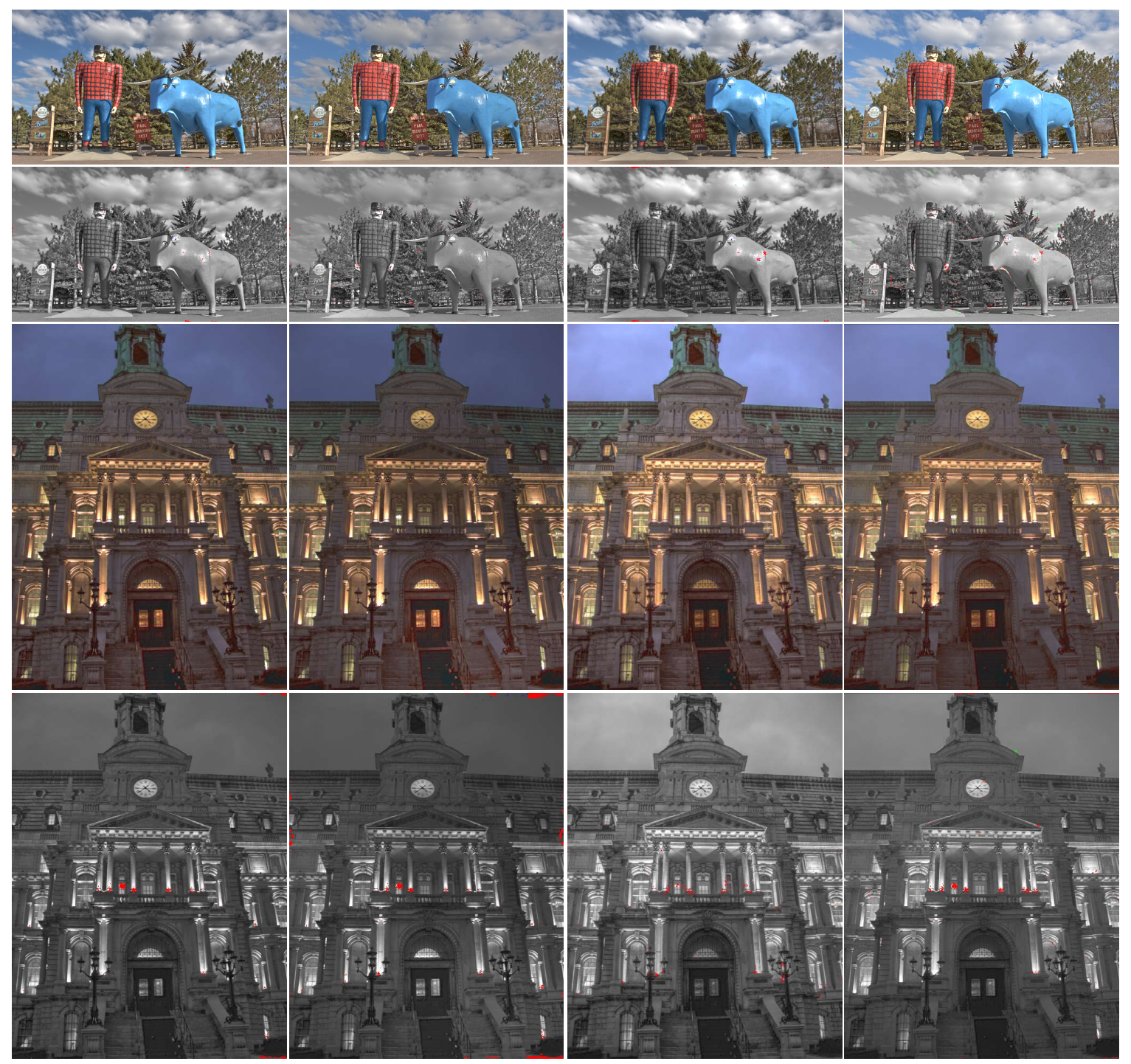

Figure 13: Output and DRIM comparison with state-of-the-art edge aware approaches. $1^{\text {st }}$ and $3^{\text {rd }}$ - rows output of the edge aware techniques; $2^{\text {nd }}$ and $4^{\text {th }}$ rows - DRIM metric [12] with probability of 95\%. Parameters used - Farbmann et al. [10] multiscale approach balanced - Fattal's [11] $\alpha=$ $0.8, \beta=0.19$ and $\gamma=0.9$; - Paris et al. [9] $\sigma_{r}=\log (2.5), \alpha=0.5$ and $\beta=0.0$ (for conveying the local effect) - The Present HOR $1^{\text {st }}$ row: $\beta=0.4, \gamma$ $=0.8 ; 3^{r d}$ row: $\beta=0.6, \gamma=0.8$. 


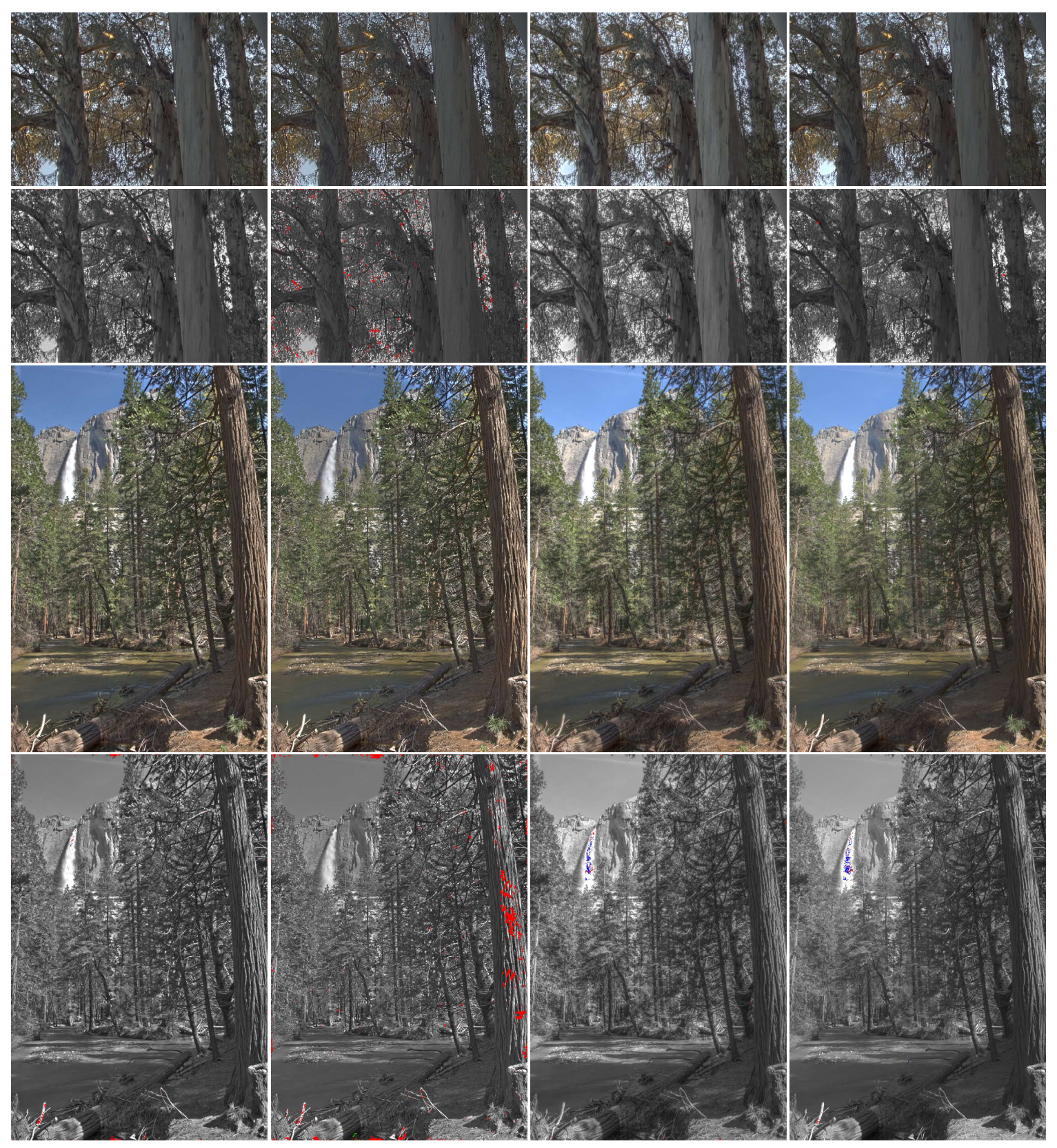

Figure 14: Output and DRIM comparison with state-of-the-art edge aware approaches. $1^{\text {st }}$ and $3^{\text {rd }}$ - rows output of the edge aware techniques; $2^{\text {nd }}$ and $4^{\text {th }}$ rows - DRIM metric [12] with probability of 95\%. Parameters used - Farbmann et al. [10] multiscale approach balanced - Fattal's [11] $\alpha=$ $0.8, \beta=0.19$ and $\gamma=0.9$; - Paris et al. [9] $\sigma_{r}=\log (2.5), \alpha=0.5$ and $\beta=0.0$ (for conveying the local effect) - The Present HOR $1^{\text {st }}$ row: $\beta=0.6, \gamma$ $=0.9 ; 3^{\text {rd }}$ row: $\beta=0.7, \gamma=0.9$. 